\title{
MUSCULOSKELETAL MANIFESTATIONS IN ACUTE LEUKEMIA: DIFFERENTIAL DIAGNOSIS IN RHEUMATOLOGY
}

Vanessa Félix Nascimento Coelho", , Thais de Campos Ferreira Pinto', María Verónica Russo Macchi', Flavia Regina Andrade

1. Pontifícia Universidade Católica de Campinas, Campinas (SP), Brazil.

*Corresponding author: vanfcoelho@yahoo.com.br

\section{BACKGROUND}

Musculoskeletal manifestations such as arthralgia, arthritis, diffuse muscle pain, myositis, associated or not with systemic symptoms - fever, weight loss, hepatosplenomegaly - are common in rheumatological diseases. However, they are also early symptoms of neoplasms, especially oncohematological ones. The association between these different diagnoses is more evident in the pediatric population. In adults, the frequency of joint symptoms is $4 \%$ in leukemias, making this situation a diagnostic challenge in clinical practice.

\section{CASE REPORT}

A 33-year-old female patient started attending a rheumatology practice, presenting a clinical state of uncharacteristic rhythm arthralgia and migratory myalgia for approximately one month. The physical examination showed no changes and the first laboratory exams showed high inflammatory markers, CRP $19.8 \mathrm{mg} / \mathrm{L}$ and ESR $53 \mathrm{~mm}$, and negative ANA and rheumatoid factor. With these findings, other exams were requested to investigate the condition. In the following visits, musculoskeletal complaints persisted. However, in these new tests, normocytic and normochromic anemia with reticulocytes of $3.4 \%$ and ferritin of $1038 \mathrm{ng} / \mathrm{mL}$ was observed, referring the patient to the hematologist, who opted for a laboratory follow-up and evaluation of bone marrow biopsy. Due to the significant worsening of symptoms, the patient sought hospital care. After initial pain management, it was decided to continue the investigation of anemia, through a bone marrow biopsy, which was positive for acute myeloid leukemia with anomalous partial expression of CD19 (Fig. 1).

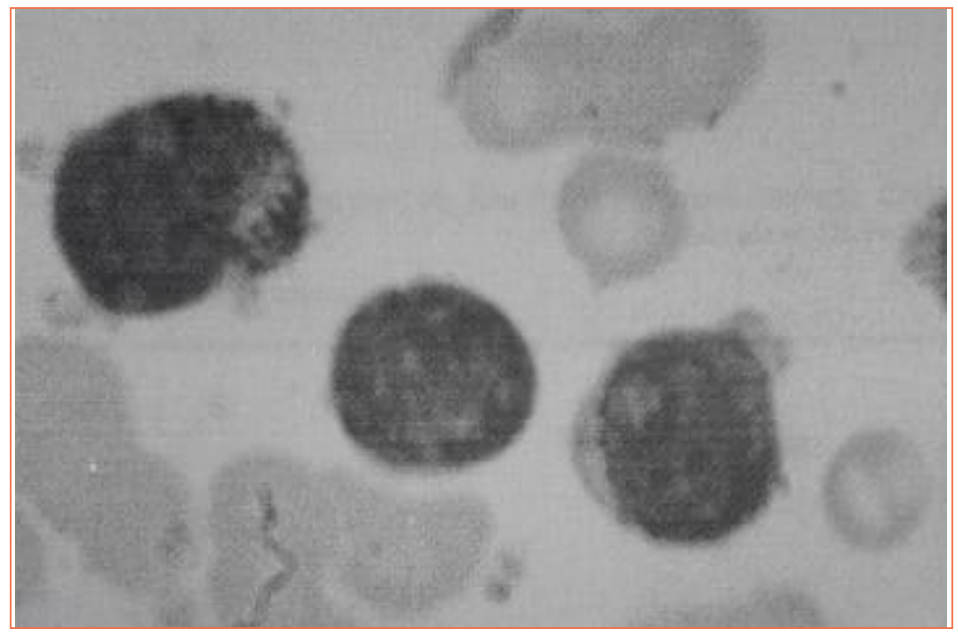

Figure 1. Bone marrow compatible with acute myeloid leukemia, with $82 \%$ of blasts. Presence of small and intermediate size blasts, high nucleus - cytoplasmic ratio, basophilic and granular cytoplasm, with some blasts showing regular cytoplasmic nucleus projections, loose chromatin and the presence of one large and evident nucleolus. 


\section{CONCLUSION}

The above report is an example of an initial picture of acute leukemia, which may involve arthralgia, asymmetric or migratory polyarthritis, often with bone pain and myalgia. Thus, atypical musculoskeletal changes, especially with negative antibodies, deserve further investigation for differential diagnoses. 